\title{
Compatibility of the nuclear shell and nucleon bag models
}

\author{
G. Krein* and Th. A. J. Maris \\ Instituto de Física, Universidade Federal do Rio Grande do Sul, 90049 Porto Alegre, R.S. Brazil
}

(Received 8 July 1986)

\begin{abstract}
It is shown that the standard self-consistency argument, reconciling the nuclear independentparticle model with the large low-energy nucleon-nucleon cross sections, loses its validity for nucleon bags with a radius larger than about $1 \mathrm{fm}$.
\end{abstract}

\section{INTRODUCTION}

For the theoretical treatment of a realistic many-body system it is necessary to introduce a model with a manageable number of degrees of freedom as a zero order approximation. In most cases the choice of such a model is suggested by the experimental observation of some qualitative properties of the system. The hope is that the more detailed properties will be given by calculable corrections to the zero order model.

Once a model has been formulated, the theory has the minimum task of showing its self-consistency: the essential assumptions of the model should not be clearly invalidated by the corrections. The present paper studies this self-consistency problem for the case of the nuclear independent-particle model with bag-like nucleons.

A good part of the successful calculations performed in nuclear physics during the last thirty years has been based on the assumption that the independent-particle model is a good zero order approximation to the nucleus. The essence of this model is the hypothesis that any particular nucleon behaves in the nucleus as a particle of a Fermi sea, moving in a smooth potential and having a mean free path which is large compared to the nuclear dimension.

Historically, the experimentally observed validity of the nuclear shell model ${ }^{1}$ was quite surprising, because the large nucleon-nucleon cross sections had earlier lead to the belief that the nucleons in a nucleus would be similar to the molecules in a liquid drop, having a very small mean free path.

In respect to the experimental situation, it should be stressed that the validity of the shell model is a much stronger statement than the existence of low energy single-particle-like excitations, which are not uncommon in Fermi systems. ${ }^{2}$ In fact, the single-particle behavior of the nucleons appears to be almost literally true. ${ }^{3}$ This behavior is, for example, evident in quasifree scattering ${ }^{4}$ and in the often quite nontrivial quantum numbers of the loosely bound nucleons in heavy nuclei. ${ }^{5}$

The compatibility of the large size of the low energy cross sections with the shell model was recognized by Weisskopf. ${ }^{6}$ He remarked that in the independent-pair model the Pauli exclusion principle drastically reduces the effects of the nucleon-nucleon interaction because the low-lying single-nucleon states are already occupied. This argument was quantitatively confirmed by means of the Bethe-Goldstone equation, ${ }^{7}$ which allows us to show that in the independent-pair approximation in nuclear matter not only the effective two-particle cross section vanishes exactly, but also a typical pair is only quite locally correlated.

With the advent of quantum chromodynamics a nucleon became to be described as a bag containing three elementary quarks. This model satisfactorily explains many properties of free hadrons and their interactions. The assumed bag radii vary between 0.3 and $1.2 \mathrm{fm}$, depending on the model considered. ${ }^{8}$ In the present paper we mean by the expression "bag" the region in which the three valence quarks of a nucleon are contained, without having any specific model in mind.

It is the purpose of the present paper to show that a nucleon bag model with a radius larger than about one fermi invalidates the usual explanation of the independent-pair approximation. We do this by trying to apply, as good as we see it possible, the usual argument for a vanishing effective cross section to nucleon pairs consisting of bag nucleons. Instead of having a vanishing cross section for the collision of a nucleon pair, we find that for bag nucleons this cross section is finite and grows fast with the assumed bag radius for bags larger than $0.7 \mathrm{fm}$, where it is still small. For a radius of about one fermi the mean free nucleon path becomes equal to the radius of a heavy nucleus.

Our argument has the typical awkwardness and limitation of many ad absurdum reasonings. One might, for example, remark that, looking at the complete $N$-body problem, the cross section of the scattering of a nucleon pair into occupied states makes no sense. It is a ground state to ground state transition which should not cause an instability of this ground state. Such a reasoning implies, however, the giving up of the two-body equation, thus invalidating the usual argument for the near locality of the two-particle correlations necessary to argue the stability of the single-particle ground state. In fact, the finite cross section we found is caused by the nonorthogonality of the two-particle-two-hole states in respect to the ground state and this will just have a delocalizing effect on the contributions of these states to the transition matrix element. We do not further follow this line of thought, as it seems that once the Bethe-Goldstone equation is not even approximately valid because of the existence of quarks degrees of freedom, one should make a fresh start, taking the quark dynamics more explicitly into account. The simple point of the present paper is to remark that for bag radii of a size often considered in the literature, the shell 
model has at present no explanation. This conclusion is in agreement with our earlier estimate of the degradation of the single-particle states in a Fermi sea of bag nucleons. ${ }^{9}$ It is hoped that these observations may stimulate the invention of a quantitative treatment of the important and perhaps somewhat neglected problem of understanding the experimentally observed validity of the independent-particle model for bag nucleons.

In Sec. II we briefly review the points of the shell model and bag models which we need for our discussion and consider an independent-pair equation for bags. The results are discussed in Sec. III and finally some remarks are made.

\section{THE INDEPENDENT-PAIR EQUATION AND BAG NUCLEONS}

\section{A. The independent-pair equation for elementary nucleons}

We first sketch the main line of thought in the formulation of the problem of the scattering of a pair of elementary nucleons in a Fermi sea.

The scattering of an isolated pair of nucleons is governed by the ordinary wave equation (with $\hbar c=1$ ):

$$
\begin{aligned}
& \left(\nabla_{1}^{2}+\nabla_{2}^{2}+\mathbf{p}_{i}^{2}+\mathbf{p}_{j}^{2}\right) \psi_{i j}\left(\mathbf{r}_{1}, \mathbf{r}_{2}\right)=J_{i j}\left(\mathbf{r}_{1}, \mathbf{r}_{2}\right), \\
& J_{i j}\left(\mathbf{r}_{1}, \mathbf{r}_{2}\right)=2 m V\left(\mathbf{r}_{1}, \mathbf{r}_{2}\right) \psi_{i j}\left(\mathbf{r}_{1}, \mathbf{r}_{2}\right),
\end{aligned}
$$

where $V\left(\mathbf{r}_{1}, \mathbf{r}_{2}\right)$ is the interaction potential and $\mathbf{p}_{i}$ and $\mathbf{p}_{j}$ are the asymptotic momenta of the two nucleons. The cross section for the scattering into the final states $\mathbf{p}_{k}$ and $\mathbf{p}_{l}$, is given by

$$
\begin{aligned}
\sigma(k l, i j) \sim \mid \int & d^{3} r_{1} d^{3} r_{2} J_{i j}\left(\mathbf{r}_{1}, \mathbf{r}_{2}\right) \\
& \times\left.\exp \left[-i\left(\mathbf{p}_{k} \cdot \mathbf{r}_{1}+\mathbf{p}_{l} \cdot \mathbf{r}_{2}\right)\right]\right|^{2},
\end{aligned}
$$

or more compactly written

$$
\sigma(k l, i j) \sim\left|\left\langle k l|V| \psi_{i j}\right\rangle\right|^{2}=|\langle k l|T| i j\rangle|^{2},
$$

where we used the definition of the $T$ matrix.

In the case of nucleons embedded in nuclear matter, Eq. (1) is no longer valid even including a mean potential and an effective-mass approximation, since the Pauli exclusion principle forbids any scattering into occupied states which therefore must be excluded. The Pauli principle can be taken into account by modifying the interaction in such a way that it vanishes for final states inside the Fermi sea. This is achieved by replacing $J_{i j}$ in Eq. (1) by

$$
J_{i j}\left(\mathbf{r}_{1}, \mathbf{r}_{2}\right)=2 m Q_{i j}\left(\mathbf{r}_{1}, \mathbf{r}_{2}\right) V\left(\mathbf{r}_{1}, \mathbf{r}_{2}\right) \psi_{i j}\left(\mathbf{r}_{1}, \mathbf{r}_{2}\right),
$$

where $Q_{i j}$ is an operator ${ }^{10}$ which allows scattering into states that are outside the Fermi sea:

$$
Q_{i j}=|i j\rangle\left\langle i j\left|+\sum_{m, n>k_{F}}\right| m n\right\rangle\langle m n|,
$$

and the cross section is now given by

$$
\sigma(k l, i j) \sim\left|\left\langle k l\left|Q_{i j} V\right| \psi_{i j}\right\rangle\right|^{2}=|A+B|^{2},
$$

where we defined $A$ and $B$ by

$$
\begin{aligned}
& A=\langle k l \mid i j\rangle\langle i j|T| i j\rangle, \\
& B=\sum_{m, n>k_{F}}\langle k l \mid m n\rangle\langle m n|T| i j\rangle,
\end{aligned}
$$

for our present purpose neglecting the modification of the $T$ matrix by many-body effects. Averaging over initial spins isospins, and summing over final momenta and spins isospins, we obtain for the average total cross section $\sigma_{i j}$ of a pair with momenta $p_{i}, p_{j}$

$$
\sigma\left(\mathbf{p}_{i}, \mathbf{p}_{j}\right)=\frac{1}{16} \sum_{t_{i} t_{f}} \sum_{m_{i} m_{f}} \sum_{\mathbf{p}_{k} \mathbf{p}_{l}} \sigma(k l, i j)
$$

The mean free path $\lambda_{i}$ for a nucleon in momentum state $i$ is

$$
\lambda_{i}=\left(\rho \sigma_{i}\right)^{-1},
$$

where $\rho$ is the average nuclear density and $\sigma_{i}$ is the average over $\mathbf{p}_{j}$ of $\left(\mathbf{p}_{i}, \mathbf{p}_{j}\right)$.

Both $A$ and $B$ in Eqs. (7) are trivially zero because of the orthogonality of two different pair states and an infinite mean free path follows.

\section{B. Modification for bag nucleons}

The equation

$$
\left\langle k l\left|Q_{i j} V\right| \psi_{i j}\right\rangle=0
$$

for $(k l \neq i j)$ and $E_{k l}=E_{i j}$, has, of course, the simple physical meaning that the projection operator $Q_{i j}$ does not allow long range scattering because the energy conserving states are occupied.

Suppose now we have bag nucleons and attempt to construct an operator $Q_{i j}^{\prime}$ corresponding to the operator $Q_{i j}$. Immediately the difficulty arises that the nucleon pair states in this case are not orthonormal. To treat this problem, we employ the formalism used in Ref. 9, which we briefly restate, in Eqs. (11)-(16), to establish the notation.

We consider a Fermi sea of $N$ noninteracting bag nucleons in a box of volume $\Omega$ with periodic boundary conditions implied, and later take the limits $N \rightarrow \infty, \Omega \rightarrow \infty$, for constant density $\rho=N / \Omega$. The ground state of this system is written as

$$
|\widetilde{0}\rangle=\prod_{i=1}^{N} B_{i}^{\dagger}|0\rangle,
$$

where $B_{i}^{\dagger}$ and $B_{i}$ are creation and annihilation operators for bag nucleons with c.m. momentum $\mathbf{P}_{i}$, spin projection $m_{i}$, and isospin projection $t_{i}$, conjunctively denoted by $i=\left\{\mathbf{P}_{i}, m_{i}, t_{i}\right\}$, with the momenta $\mathbf{P}_{i}$ assuming the lowest possible values consistent with the Pauli principle as applied to point nucleons.

For a nucleon creation operator we write

$$
B_{i}^{\dagger}=C_{i}^{\mu_{1} \mu_{2} \mu_{3}} q_{\mu_{1}}^{1 \dagger} q_{\mu_{2}}^{2 \dagger} q_{\mu_{3}}^{3 \dagger},
$$

where in the quark creation operators $q^{\dagger}$,s the upper indices 1,2 , and 3 denote color and the lower indices $\mu$ 's denote momentum and spin-isospin projection of the 
quarks in the totally symmetrical nucleon wave functions $C_{i}^{\mu_{1} \mu_{2} \mu_{3}}$. These $C$ 's are chosen such that the one-nucleon states are normalized to one.

Instead of the usual anticommutation rules for the point nucleons case, now we have

$$
\left\{B_{i}, B_{j}^{\dagger}\right\}=\delta_{i j}-A_{i j}, \quad\left\{B_{i}, B_{j}\right\}=\left\{B_{i}^{\dagger}, B_{j}^{\dagger}\right\}=0,
$$

where

$$
A_{i j}=C_{i}^{\mu_{1} \mu_{2} \mu_{3}} C_{j}^{\mu_{1}^{\prime} \mu_{2}^{\prime} \mu_{3}^{\prime} *}\left[\delta_{\mu_{1}^{\prime} \mu_{1}} \delta_{\mu_{2}^{\prime} \mu_{2}} q_{\mu_{3}}^{3^{\dagger}} q_{\mu_{3}^{\prime}}^{3}-\delta_{\mu_{1}^{\prime} \mu_{1}} q_{\mu_{2}}^{2 \dagger} q_{\mu_{2}^{\prime}}^{2} q_{\mu_{3}}^{3 \dagger} q_{\mu_{3}^{\prime}}^{3}+\operatorname{cycl} .(1,2,3)\right]
$$

where the anticommutation rules for the quark operators were used.

The analogues of the two-particle states occurring in the Bethe-Goldstone equation are given by

$$
|k l\rangle=B_{k}^{\dagger} B_{l}^{\dagger}|0\rangle \text {. }
$$

Using the anticommutation rules of Eqs. (13), one obtains

$$
\langle k l \mid m n\rangle=\delta_{k m} \delta_{l n}-\delta_{k n} \delta_{l m}-\Delta(k l, m n),
$$

where

$$
\Delta(k l, m n)=3 C_{k}^{\mu_{1} \mu_{2} \mu_{3} *} C_{m}^{\mu_{1} \mu_{2} v_{3}} C_{l}^{\sigma_{1} \sigma_{2} v_{3} *} C_{n}^{\sigma_{1} \sigma_{2} \mu_{3}}-(m \leftrightarrow n) .
$$

\section{Defining $Q_{i j}^{\prime}$ by}

$$
Q_{i j}^{\prime}=|i j\rangle\left\langle i j\left|+\sum_{m, n>k_{F}}\right| m n\right\rangle\langle m n|,
$$

where $|i j\rangle$ and $|m n\rangle$ are two bag-nucleon states in the Fermi sea, Eq. (11), we find that this operator, in contradistinction to $Q_{i j}$ of Eq. (5), is not anymore a perfect projection operator, because of the term $\Delta(k l, m n)$ on the right-hand side of Eq. (15a). The nonorthogonality of the two-particle states and the nonprojectivity of $Q_{i j}^{\prime}$ have a physical origin. The two-particle-two-hole states are not anymore eigenstates of a zero order Hamiltonian, because of the new quark degrees of freedom. There exists a state space outside the nucleon space which may seriously affect the Bethe-Goldstone equation and its consequences.

As a remedy for this trouble does not seem to be known, let us attempt to estimate the damage caused by the nonprojectivity of $Q_{i j}^{\prime}$. Inserting Eq. (15b) into Eqs. (7), one obtains

$$
\begin{aligned}
A^{\prime} & =-\Delta(k l, i j)\langle i j|T| i j\rangle, \\
B^{\prime} & =-\sum_{m, n>k_{F}} \Delta(k l, m n)\langle m n|T| i j\rangle .
\end{aligned}
$$

To calculate these integrals we need the explicit form of the quark wave functions of Eq. (12), which we take as in Ref. 9:

$$
\begin{aligned}
& B_{i}^{\dagger}= C_{i}^{\mu_{1} \mu_{2} \mu_{3}} q_{\mu_{1}}^{1^{\dagger}} q_{\mu_{2}}^{2 \dagger} q_{\mu_{3}}^{3 \dagger} \\
&=N_{i} \sum_{\mathbf{k}^{\prime} s} \sum_{\alpha^{\prime} s} \delta_{\mathbf{k}_{1}+\mathbf{k}_{2}+\mathbf{k}_{3}}^{\mathbf{P}_{i}} \phi\left(\mathbf{k}_{1}\right) \phi\left(\mathbf{k}_{2}\right) \phi\left(\mathbf{k}_{3}\right) \\
& \\
& \quad \times(18)^{-1 / 2} T_{i}^{\alpha_{1} \alpha_{2} \alpha_{3}} q_{\alpha_{1}}^{1^{\dagger}}\left(\mathbf{k}_{1}\right) q_{\alpha_{2}}^{2 \dagger}\left(\mathbf{k}_{2}\right) q_{\alpha_{3}}^{3^{\dagger}}\left(\mathbf{k}_{3}\right)
\end{aligned}
$$

where each of the $\alpha_{i}$ 's specifies one of the four quark spin-isospin states, the $T_{i}^{\alpha_{1} \alpha_{2} \alpha_{3}}$ represents the generalized Clebsch-Gordan coefficients ${ }^{11}$ for our case, $N_{i}$ is a normalization constant, and the $\delta$ function guarantees the exact c.m. momentum $\mathbf{P}_{i}$ for the nucleon. As stated earlier, we take for the single quark momentum wave functions

$$
\phi(\mathbf{k})=\left(r^{2} / \pi\right)^{3 / 4} \exp \left(-\frac{1}{2} r^{2} \mathbf{k}^{2}\right)
$$

where $r$ is the rms radius of the quark distribution of the nucleon, with the c.m. correction included.

With these wave functions, we obtain, for $\Delta(k l, m n)$ in Eq. (15b),

$$
\begin{aligned}
\Delta(k l, m n)= & 3\left(3 r^{2} / 4 \pi\right)^{3 / 2}\left(8 \pi^{3} / \Omega\right) \delta_{\mathbf{p}_{k}+\mathbf{p}_{l}, \mathbf{p}_{m}+\mathbf{p}_{n}} \\
& \times \chi(k l, m n) \exp \left[\left(-\frac{7}{12} \mathbf{p}_{k}^{2}+\frac{1}{6} \mathbf{p}_{l}^{2}-\frac{1}{4} \mathbf{p}_{n}^{2}+\frac{1}{2} \mathbf{p}_{k} \cdot \mathbf{p}_{l}+\mathbf{p}_{k} \cdot \mathbf{p}_{n}-\frac{1}{3} \mathbf{p}_{m} \cdot \mathbf{p}_{n}\right) r^{2}\right]-(m \leftrightarrow n),
\end{aligned}
$$

where $\chi(k l, m n)$ is given by

$\chi(k l, m n)=(18)^{-2} T_{k}^{\alpha_{1} \alpha_{2} \alpha_{3}} T_{m}^{\alpha_{1} \alpha_{2} \beta_{3}} T_{l}^{\gamma_{1} \gamma_{2} \beta_{3}} T_{n}^{\gamma_{1} \gamma_{2} \alpha_{3}}$.

As shown in Appendix A, from Eqs. (20), follows that, in the limits $N \rightarrow \infty, \Omega \rightarrow \infty$ with $\rho=N / \Omega$ constant, the contribution to the pair cross section coming from the term corresponding to $A$ in Eq. (7a) remains zero but the contribution of $B^{\prime}$ does not vanish anymore. We therefore have, instead of Eq. (6)

$$
J(k l, i j) \sim\left|B^{\prime}\right|^{2} .
$$

The basic reason for this different behavior is that in Eq. (7a) for $A$ only one intermediate state contributes, in contradistinction to the infinite sum occurring in Eq. (7b).

\section{The mean free path}

For the present crude estimate we choose in Eqs. (17) an effective expression for $T$ such that $\langle m n|T| i j\rangle$ reproduces the experimental average total $s$-wave cross section for free nucleon-nucleon scattering. We take for the scattering matrix element 
$\langle r s|T| i j\rangle=V_{0} / \Omega\left(\pi a^{2}\right)^{3 / 2} \delta_{\mathbf{p}_{r}+\mathbf{p}_{s}, \mathbf{p}_{i}+\mathbf{p}_{j}}\left\{\delta_{r i} \delta_{s j} \exp \left[-\left(\mathbf{p}_{r}-\mathbf{p}_{i}\right)^{2} a^{2} / 4\right]-(i \leftrightarrow j)\right\}$,

where $^{11} V_{0}=0.25 \mathrm{fm}^{-1}$ and $a=2 \mathrm{fm}$ are, respectively, the depth and the range of the effective potential and $\delta_{r i}$ and $\delta_{s j}$ are spin-isospin Kronecker deltas.

In order to obtain a typical value for the mean free path we choose for $\sigma_{i}$ in Eq. (9) the cross section for nucleons with initial momenta which are equal and opposite $\mathbf{p}_{i}=-\mathbf{p}_{j}$, with $\left|\mathbf{p}_{i}\right|=k$ equal to the rms momentum in the Fermi distribution. Inserting the value of $B^{\prime}$, it is shown in Appendix B that we obtain from Eq. (8) for the cross section

$$
\begin{aligned}
\sigma= & \pi / 24\left(m^{*} V_{0} a^{2}\right)^{2}(3 r)^{6} \\
& \times \exp \left[-\left(\frac{5}{6} r^{2}+\frac{1}{2} a^{2}\right) k^{2}\right] F\left(k_{F}, r\right),
\end{aligned}
$$

where $F\left(k_{F}, r\right)$ is a function, defined in Appendix B, which depends on the Fermi momentum $k_{F}$ and on the rms radius $r$ of the quark distribution and $m^{*}$ is the effective mass in nuclear matter.

The results of the calculation for the cross section $\sigma$ and the mean free path $\lambda=(\rho \sigma)^{-1}$ are shown by the solid lines in Figs. 1 and 2, respectively, with the effective mass chosen to be 0.65 times the proton mass. ${ }^{12}$ Basically because of the steepness of the mean free path and cross section curves, our conclusions are not sensitively dependent on this value. The dotted, dashed, and dotted-dashed curves in Figs. 1 and 2 represent the results of the modified calculations discussed in Sec. II D.

\section{Modified calculations}

In order to investigate the dependence of the results on the shape of the nucleon wave functions, we also performed the calculations for other choices of the radial

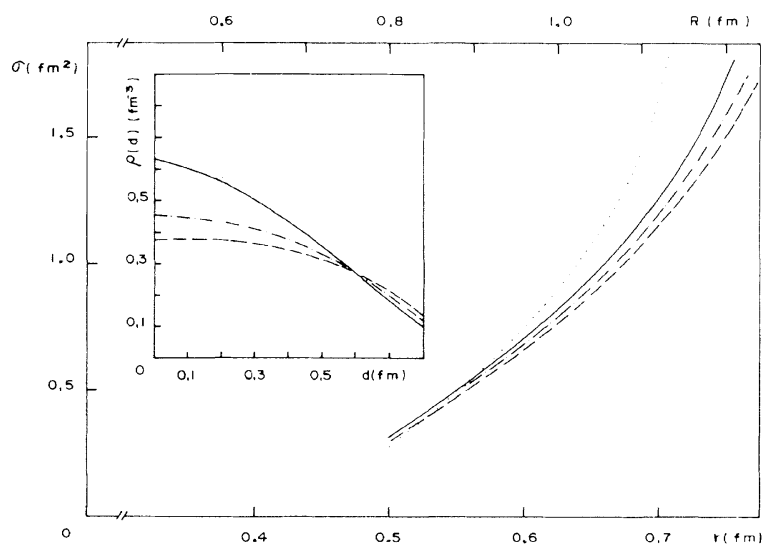

FIG. 1. The average cross section for a typical nucleon in nuclear matter of normal density as a function of the rms radius of the quark distribution (lower scale) and of the equivalent bag radius (Ref. 18) $R=1.6 r$ (upper scale). The solid, dotted-dashed, and dashed curves correspond, respectively, to $\epsilon=0, \epsilon=0.25$, and $\epsilon=0.5$. In the inset, quark densities as functions of the distance $d$ from the center of the bag, for the values of above, all with the same rms radius of $0.8 \mathrm{fm}$. The dotted curve corresponds to a different choice of the operator $Q_{i j}^{\prime}$, Eq. (27). dependence of the quark wave functions.

Introducing center-of-mass and relative coordinates

$$
\begin{aligned}
& \mathbf{R}=\frac{1}{3}\left(\mathbf{r}_{1}+\mathbf{r}_{2}+\mathbf{r}_{3}\right), \\
& \xi=1 / \sqrt{2}\left(\mathbf{r}_{1}-\mathbf{r}_{2}\right), \\
& \eta=1 / \sqrt{6}\left(\mathbf{r}_{1}+\mathbf{r}_{2}-2 \mathbf{r}_{3}\right)
\end{aligned}
$$

in Eq. (18) for the quark wave functions one finds

$$
\begin{aligned}
\psi_{\mathbf{P}}(\mathbf{R}, \boldsymbol{\xi}, \boldsymbol{\eta}) & =(\Omega)^{-1 / 2} e^{i \mathbf{P} \cdot \mathbf{R}} \boldsymbol{\Phi}_{\text {int }}(\boldsymbol{\xi}, \boldsymbol{\eta}) \\
& =(\Omega)^{-1 / 2} e^{i \mathbf{P} \cdot \mathbf{R}}\left(\pi r^{2}\right)^{-3 / 2} e^{-\left(\xi^{2}+\eta^{2}\right) / 2 r^{2}} .
\end{aligned}
$$

The internal wave function $\Phi_{\text {int }}$ was modified to give more uniform quark density distributions inside the bag:

$$
\begin{aligned}
\Psi_{\mathbf{P}}(\mathbf{R}, \xi, \boldsymbol{\eta})= & (\Omega)^{-1 / 2} e^{i \mathbf{P} \cdot \mathbf{R}} \\
& \times C(\epsilon)\left[\Phi_{\text {int }}^{(1)}(\xi, \boldsymbol{\eta})-\epsilon \Phi_{\text {int }}^{(2)}(\xi, \boldsymbol{\eta})\right],
\end{aligned}
$$

where $\Phi_{\text {int }}^{(1)}$ and $\Phi_{\text {int }}^{(2)}$ are of the same Gaussian type of $\Phi_{\text {int }}$ in Eq. (25), but with different individual widths, $r_{1}$ and $r_{2}$; the parameter $\epsilon$ is varied and $C(\epsilon)$ is chosen to keep the normalization constant. For $\epsilon=0$, one obtains the previously used wave function.

The results obtained with the wave functions of Eq. (25) and two different shapes of Eq. (26), are shown by the solid and dashed and dotted-dashed curves, respectively,

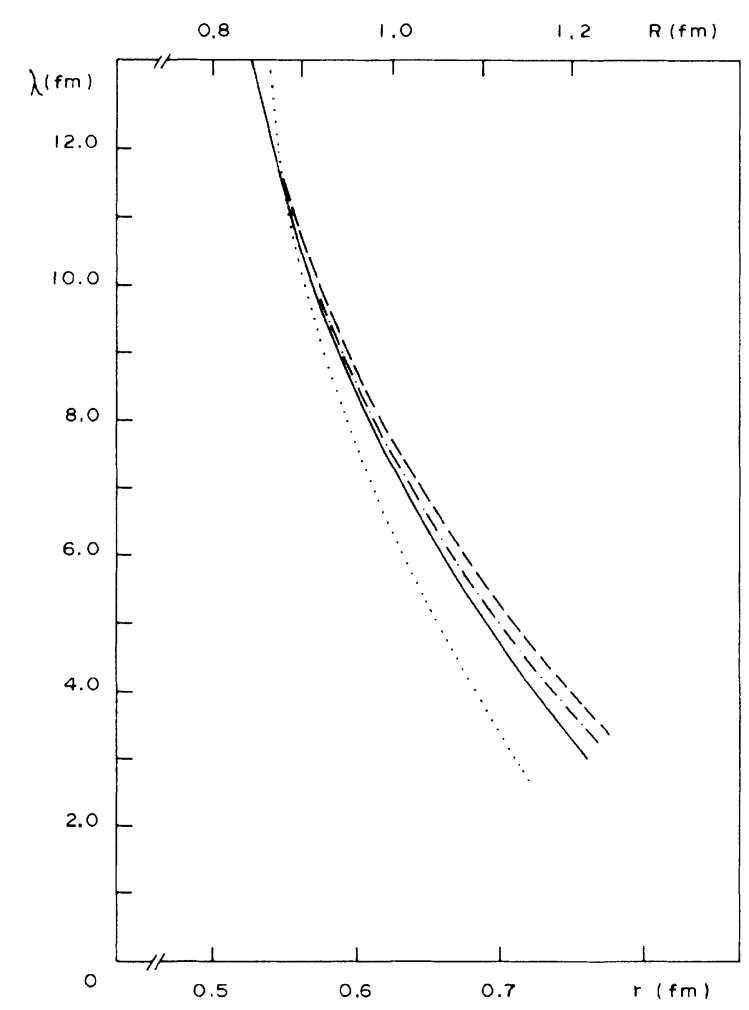

FIG. 2. The mean free paths corresponding to the cross sections of Fig. 1. 
in Figs. 1 and 2. Shown in the inset of Fig. 1 are the quark densities for $\epsilon=0$ and $r=0.8 \mathrm{fm}$ (solid lines), $\epsilon=0.5$ and $r_{1}=0.7 \mathrm{fm}, r_{2}=0.5 \mathrm{fm}$ (dashed lines), and $\epsilon=0.3$ and $r_{1}=0.75 \mathrm{fm}, r_{2}=0.4 \mathrm{fm}$ (dotted-dashed lines), all giving the same rms radius of $0.8 \mathrm{fm}$. As can be seen, there is no substantial sensitivity of the results on the shape of the wave functions.

Another observation which can be made on our calculations is the following. Because the "projection operator" $Q_{i j}^{\prime}$ which we use in the independent-pair equation is not anymore exact for composed nucleons, identities between projection operators for point nucleons are not anymore identities for the corresponding operators $Q_{i j}^{\prime}$ in the case of extended bags. It is then not anymore indifferent in which form the exact operators, which we translate into our picture, are written. In practice, this arbitrariness seems not to make much difference in the results.

As an example, we have calculated the cross section by writing for $Q_{i j}^{\prime}$, instead of the expression of Eq. (16), the, for point nucleons identical, expression

$$
Q_{i j}^{\prime}=|i j\rangle\left\langle i j\left|+1-\sum_{m, n<k_{F}}\right| m n\right\rangle\langle m n| .
$$

Instead of only allowing states outside the Fermi sea for the scattering, this formulation excludes the states inside the Fermi sea.

The calculation for this case has a rather different appearance from the previous one, but the results, for the wave functions of Eq. (25), indicated by the dotted curves in the figures, are evidently quite similar.

\section{DISCUSSION AND CONCLUDING REMARKS}

Figure 2 shows that, for a confinement radius up to 0.7 $\mathrm{fm}$, the estimated mean free path is such that the arguments on the basis of the Bethe-Goldstone equation for the shell model are not invalidated in the case of composite nucleons, in agreement with the general expectation. ${ }^{14}$ But for radii larger than one fermi the mean free path obtained following these arguments become equal or smaller than the radius of a heavy nucleus and one would not anymore expect the shell model to work.

The use of the concept of the mean free path of a bound nucleon may not be generally accepted, although it has been regularly used in the literature. ${ }^{15,16}$ If desired, one may translate the value of the mean free path or of the average total cross section, via the optical theorem, into the value of an imaginary shell model potential, which may not be too large for the shell model to be valid.

The nonorthogonality of the two-particle states, caused by the quark statistics operating in overlapping nucleons, implies the presence of quark configurations which tend to have an increased kinetic energy. ${ }^{17}$ This effect may lead to a repulsion in the nucleon-nucleon interaction, as has indeed been observed for free nucleons. Such a repulsive core, if sufficiently hard, will affect our lowest order treatment, which is based on the overlapping of undeformed nucleon wave functions. It is however well known ${ }^{7}$ that a strongly repulsive core in the nucleon-nucleon interaction must really remain small in order not to destroy by itself the selfconsistency of the independent-pair approximation. As long as the effective volume of such a core is small compared to the volume of a bag with the critical radius of about one fermi, our estimates will not be radically modified.

Recently, ${ }^{19}$ the interesting remark has been made that the antisymmetrization of the quark wave functions for a shell model with 6-quark bag correlations does not necessarily invalidate certain predictions of the original shell model. This question differs from the one with which the present paper is concerned, namely, how to justify the relevance of single-particle states, as, for instance, used in the just mentioned calculation.

As has been already remarked, our calculation can only give a kind of first order deviation from the usual independent-pair equation. This avoids the quark interactions which would introduce, at the present stage of our knowledge, a large complication and arbitrariness. Although therefore our treatment is quite limited, as it contains almost no dynamics, it has the virtue of not being sensitively dependent on the details of bag models and quark interactions. Of course, we cannot maintain that even for radii larger than one fermi there cannot exist another good argument for the observed single-particle behavior in nuclei. However, such an explanation seems not yet to have been given and is almost certainly nontrivial. For a hybrid soliton-bag model, ${ }^{20}$ for example, nonlocal soliton-bag commutators presumably would play the part of our bag commutators, Eqs. (13).

In the present situation, the only conclusion we can draw with confidence is that the usual self-consistency argument for the independent-particle model for point nucleons might well be applicable to bags with a radius smaller than $0.7 \mathrm{fm}$, but the same reasoning throws serious doubts on the validity of the independent-particle model for bags larger than about one fermi. An understanding of the intermediate region would require the introduction of detailed dynamical assumptions. Clearly there is a need for a better treatment of this problem, namely to relate the vast experience obtained in nuclear physics to an important model in elementary particle physics. Such a study might lead to severe conditions to be obeyed by the underlying models.

\section{ACKNOWLEDGMENTS}

The authors would like to thank M. Betz and G. A. Miller for discussions. This work was supported in part by Conselho Nacional de Desenvolvimento Científico e Tecnológico (CNPq), Financiadora de Estudos e Projetos (FINEP) and Coordenação de Aperfeiçoamento de Pessoal do Ensino Superior (CAPES) (Brazilian agencies).

\section{APPENDIX A}

Here we shall show explicitly that the contribution of $A^{\prime}$ to the cross section vanishes. We begin with the wellknown expression for the cross section: ${ }^{11}$ 


$$
\sigma\left(\mathbf{p}_{i}, \mathbf{p}_{j}\right)=\frac{2 \pi}{v_{\text {rel }}} \frac{1}{16} \sum_{\substack{\text { spins } \\ \text { isospins }}} \lim _{\Omega \rightarrow \infty} \Omega \sum_{\mathbf{p}_{k} \mathbf{p}_{l}}|\langle k l|T| i j\rangle|^{2} \delta\left(E_{f}-E_{i}\right),
$$

where $v_{\text {rel }}$ is the relative velocity. Using Eq. (6), one has

$$
\sigma\left(\mathbf{p}_{i}, \mathbf{p}_{j}\right)=\frac{2 \pi}{v_{\text {rel }}} \frac{1}{16} \sum_{\substack{\text { spins } \\ \text { isospins }}} \lim _{\Omega \rightarrow \infty} \Omega \sum_{\mathbf{p}_{k} \mathbf{p}_{l}}\left|\langle k l \mid i j\rangle\langle i j|T| i j\rangle+\sum_{m, n>k_{F}}\langle k l \mid m n\rangle\langle m n|T| i j\rangle\right|^{2} \delta\left(E_{f}-E_{i}\right) .
$$

The matrix element $\langle m n|T| i j\rangle$ of Eq. (22) can be written as

$$
\langle m n|T| i j\rangle=1 / \Omega \delta_{\mathbf{p}_{m}+\mathbf{p}_{n}, \mathbf{p}_{i}+\mathbf{p}_{j}} \Gamma(m n, i j)
$$

with

$$
\Gamma(m n, i j)=\left(\pi a^{2}\right)^{3 / 2} V_{0} \delta_{m i} \delta_{n j} \exp \left[-\left(\mathbf{p}_{m}-\mathbf{p}_{i}\right)^{2} a^{2} / 4\right]-(i \leftrightarrow j)
$$

From Eqs. (20), we can write for $\langle k l \mid m n\rangle$ :

$$
\langle k l \mid m n\rangle=8 \pi^{3} / \Omega \delta_{\mathbf{p}_{m}+\mathbf{p}_{n}, \mathbf{p}_{k}+\mathbf{p}_{l} \Lambda(k l, m n),}
$$
where

$$
\Lambda(k l, m n)=3\left(3 r^{2} / 4 \pi\right)^{3 / 2} \chi(k l, m n) \exp \left[\left(-\frac{7}{12} \mathbf{p}_{k}^{2}+\frac{1}{6} \mathbf{p}_{l}^{2}-\frac{1}{4} \mathbf{p}_{m}^{2}-\frac{1}{2} \mathbf{p}_{n}^{2}+\frac{1}{2} \mathbf{p}_{k} \cdot \mathbf{p}_{m}+\mathbf{p}_{k} \cdot \mathbf{p}_{n}-\frac{1}{3} \mathbf{p}_{m} \cdot \mathbf{p}_{n}\right) r^{2}\right]-(m \leftrightarrow n)
$$

Substituting Eqs. (A3) and (A5) in Eq. (A2), we obtain

$$
\begin{aligned}
\sigma\left(\mathbf{p}_{i}, \mathbf{p}_{j}\right)=\frac{1}{(2 \pi)^{2} v_{\text {rel }}} \frac{1}{16} \sum_{\substack{\text { spins } \\
\text { isospins }}} \lim _{\Omega \rightarrow \infty}\left[\left(8 \pi^{3} / \Omega\right) \sum_{\mathbf{p}_{k}}\left(8 \pi^{3} / \Omega\right)\right. & \sum_{\mathbf{p}_{l}}\left(\Omega / 8 \pi^{3}\right) \delta_{\mathbf{p}_{k}+\mathbf{p}_{l}, \mathbf{p}_{i}+\mathbf{p}_{j}} \delta\left(E_{f}-E_{i}\right) \\
\times \mid\left(8 \pi^{3} / \Omega\right) \Lambda(k l, i j) \Gamma(i j, i j) & +\left(8 \pi^{3} / \Omega\right) \sum_{\mathbf{p}_{m}\left(8 \pi^{3} / \Omega\right)} \sum_{\mathbf{p}_{n}} \theta\left(\left|\mathbf{p}_{m}\right|-k_{F}\right) \theta\left(\left|\mathbf{p}_{n}\right|-k_{F}\right) \\
& \times\left(\Omega / 8 \pi^{3}\right) \delta_{\mathbf{p}_{m}+\mathbf{p}_{n}, \mathbf{p}_{i}+\mathbf{p}_{j}} \\
& \left.\times\left.\Lambda(k l, m n) \Gamma(m n, i j)\right|^{2}\right] .
\end{aligned}
$$

Now taking for fixed $N / \Omega=\rho$ the limits $N \rightarrow \infty, \Omega \rightarrow \infty$ :

$$
\begin{aligned}
& \left(8 \pi^{3} / \Omega\right) \sum_{\mathbf{p}} f_{\mathbf{p}} \rightarrow \int d^{3} p f(\mathbf{p}), \\
& \left(\Omega / 8 \pi^{3}\right) \delta_{\mathbf{p}} \rightarrow \delta^{(3)}(\mathbf{p}),
\end{aligned}
$$

we find for the total cross section

$$
\begin{aligned}
\sigma\left(\mathbf{p}_{i}, \mathbf{p}_{j}\right)=\frac{1}{(2 \pi)^{2} v_{\text {rel }}} \frac{1}{16} \sum_{\substack{\text { spins } \\
\text { isospins }}} \int d^{3} p_{k} d^{3} p_{l} \delta^{(3)}\left(\mathbf{p}_{k}+\mathbf{p}_{l}-\mathbf{p}_{i}-\mathbf{p}_{j}\right) \delta\left(E_{f}-E_{i}\right) \\
\times \mid\left(8 \pi^{3} / \Omega\right) \Lambda(k l, i j) \Gamma(i j, i j)+\int d^{3} p_{m} d^{3} p_{n} \theta\left(\left|\mathbf{p}_{m}\right|-k_{F}\right) \theta\left(\left|\mathbf{p}_{n}\right|-k_{F}\right) \delta^{(3)} \\
\times\left.\left(\mathbf{p}_{m}+\mathbf{p}_{n}-\mathbf{p}_{i}-\mathbf{p}_{j}\right) \Lambda(k l, m n) \Gamma(m n, i j)\right|^{2}
\end{aligned}
$$

From Eq. (A8), in the infinite volume limit, we can see that the term $A^{\prime}$ does not contribute to the cross section.

\section{APPENDIX B}

Here we shall obtain result Eq. (23). Using Eqs. (A4) and (A6) in Eq. (A8), one has

$$
\begin{aligned}
\sigma\left(\mathbf{p}_{i}, \mathbf{p}_{j}\right)=\frac{1}{(2 \pi)^{2} v_{\text {rel }}} \frac{1}{16} \sum_{\text {spons }} \int d^{3} p_{k} d^{3} p_{l} \delta^{(3)}\left(\mathbf{p}_{k}+\mathbf{p}_{l}-\mathbf{p}_{i}-\mathbf{p}_{j}\right) \delta\left(1 / 2 m^{*}\left(\mathbf{p}_{k}^{2}+\mathbf{p}_{l}^{2}-\mathbf{p}_{i}^{2}-\mathbf{p}_{j}^{2}\right)\right) & \\
\times \mid 3\left(3 r^{2} / 4 \pi\right)^{3 / 2}\left(\pi a^{2}\right)^{3 / 2} V_{0} \int & d^{3} p_{m} d^{3} p_{n} \theta\left(\left|\mathbf{p}_{m}\right|-k_{F}\right) \theta\left(\left|\mathbf{p}_{n}\right|-k_{F}\right) \delta^{(3)}\left(\mathbf{p}_{m}+\mathbf{p}_{n}-\mathbf{p}_{i}-\mathbf{p}_{j}\right) \\
& \times\left\{\chi ( k l , m n ) \operatorname { e x p } \left[\left(-\frac{7}{12} \mathbf{p}_{k}^{2}+\frac{1}{6} \mathbf{p}_{l}^{2}-\frac{1}{4} \mathbf{p}_{m}^{2}-\frac{1}{2} \mathbf{p}_{n}^{2}\right.\right.\right. \\
& \left.\left.\left.+\frac{1}{2} \mathbf{p}_{k} \cdot \mathbf{p}_{m}+\mathbf{p}_{k} \cdot \mathbf{p}_{n}-\frac{1}{3} \mathbf{p}_{m} \cdot \mathbf{p}_{n}\right) r^{2}\right]-(m \leftrightarrow n)\right\} \\
& \times\left.\left\{\delta_{m i} \delta_{n i} \exp \left[-\left(\mathbf{p}_{m}-\mathbf{p}_{i}\right)^{2} a^{2} / 4\right]-(i \leftrightarrow j)\right\}\right|^{2}, \quad(B 1)
\end{aligned}
$$


for initial momenta $\mathbf{p}_{i}=-\mathbf{p}_{j}$ and $\left|\mathbf{p}_{i}\right|=k$, Eq. (B1) becomes

$$
\sigma=\pi / 24\left(m^{*} V_{0} a^{3}\right)^{2}(3 r)^{6} \exp \left[-\left(\frac{5}{6} r^{2}+\frac{1}{2} a^{2}\right) \mathbf{k}^{2}\right] F\left(k_{F}, r\right),
$$

$M(x)$ is given by

$$
M(x)=[I(x)]^{2}-0.6 I(x) I(-x),
$$

where $I(x)$ is the following integral:

$$
I(x)=\frac{1}{2} A^{-1}(x) \int_{k_{F}}^{\infty} d p p \exp \left[-\left(\frac{5}{12} r^{2}+\frac{1}{4} a^{2}\right) p^{2}\right] \sinh [p A(x)], \quad A(x)=\frac{1}{2}\left(r^{4}+a^{4}+2 r^{2} a^{2} x\right)^{1 / 2} .
$$

From this follows Eq. (23), with $F\left(k_{F}, r\right)$ given by

$$
F\left(k_{F}, r\right)=\int_{-1}^{+1} d x M(x) \text {. }
$$

*Permanent address: Departamento de Física, Universidade Federal de Santa Maria, 97119 Santa Maria, R.S., Brazil.

${ }^{1}$ O. Haxel, J. H. D. Jensen, and H. E. Suess, Phys. Rev. 75, 1766 (1949); M. G. Mayer, Phys. Rev. 75, 1969 (1949).

${ }^{2}$ A. B. Migdal, Theory of Finite Fermi Systems and Applications to Atomic Nuclei (Wiley-Interscience, New York, 1967).

${ }^{3}$ D. Wilkinson, Comm. Nucl. Part. Phys. 2, 83 (1968).

${ }^{4}$ G. Jacob and Th. A. J. Maris, Rev. Mod. Phys. 38, 121 (1966); 45, 6 (1973).

5J. M. Cavedon et al., Phys. Rev. Lett. 40, 978 (1982); B. Frois and J. M. Laget, La Recherche 14, 1370 (1983).

${ }^{6}$ V. F. Weisskopf, Science 113, 101 (1951).

${ }^{7}$ L. C. Gomes, J. D. Walecka, and V. F. Weisskopf, Ann. Phys. (N.Y.) 3, 241 (1958).

${ }^{8}$ A. Chodos, R. L. Jaffe, K. Johnson, C. B. Thorn, and V. F. Weisskopf, Phys. Rev. D 9, 3471 (1974); W. A. Bardeen, M. S. Chanowitz, S. D. Drell, M. Weinstein, and T. M. Yan, ibid. 11, 1094 (1975); R. Friedberg and T. D. Lee, ibid. 16, 1096 (1977); G. E. Brown and M. Rho, Phys. Lett. 82B, 177 (1979); S. Théberge, A. W. Thomas, and G. A. Miller, Phys. Rev. D 22, 2838 (1980). For a review see G. A. Miller, Int. Rev. Nucl. Phys. 1 (1985).
${ }^{9}$ M. Betz, G. Krein, and Th. A. J. Maris, Nucl. Phys. A437, 509 (1985).

${ }^{10}$ H. A. Bethe and J. Goldstone, Proc. R. Soc. (London) Ser. A 238, 551 (1951).

${ }^{11}$ T. D. Lee, Particle Physics and Introduction to Field Theory (Harwood, New York, 1981).

${ }^{12}$ P. Ring and P. Schuck, The Nuclear Many-Body Problem (Springer-Verlag, New York, 1980).

${ }^{13}$ M. Brittan, Phys. Lett. 79B, 27 (1978).

${ }^{14}$ L. S. Celenza, A. Harindranath, A. Rosenthal, and C. M. Shakin, Phys. Rev. C 31, 946 (1985).

${ }^{15}$ J. M. Blatt and V. F. Weisskopf, Theoretical Nuclear Physics (Wiley, New York, 1952).

${ }^{16}$ C. Mahaux, P. F. Bortignon, R. A. Broglia, and C. H. Dasso, Phys. Rep. 120, 1 (1985).

${ }^{17}$ M. Oka and K. Yazaki, Prog. Theor. Phys. 66, 556 (1981); 66, 572 (1981). For a complete list of references see K. Maltmann and N. Isgur, Phys. Rev. D 29, 952 (1984).

${ }^{18}$ M. Betz and R. Goldflam, Phys. Rev. D 28, 284 (1983).

${ }^{19}$ G. A. Miller, Phys. Lett. 174B, 229 (1986).

${ }^{20}$ M. Rho, A. Goldhaber, and G. E. Brown, Phys. Rev. Lett. 51, 747 (1983). 